\title{
A Non-Interventional Multicenter Study of First-Line Bevacizumab in Combination with Chemotherapy in Patients with Metastatic Colorectal Cancer in Lebanon
}

\author{
Sally Temraz $\mathbb{D}^{1}$, Fadi Nasr ${ }^{2}$, Joseph Kattan ${ }^{3}$, Dany Abigerges ${ }^{4}$, Walid Moukadem ${ }^{5}$, Fadi Farhat $\mathbb{D}^{6}$, \\ Layal Maatouk ${ }^{7}$, Georges Chahine ${ }^{8}$, Ali Shamseddine $\mathbb{D}^{1}$ \\ 'Department of Internal Medicine, American University of Beirut Medical Center, Beirut, Lebanon; ${ }^{2}$ Department of Hematology and Oncology, Mount \\ Lebanon Hospital, Beirut, Lebanon; ${ }^{3}$ Department of Hematology and Oncology, Saint- Joseph University, Beirut, Lebanon; ${ }^{4}$ Department of \\ Hematology and Oncology, Middle East Institute of Health, Bsalim, Lebanon; ${ }^{5}$ Department of Hematology and Oncology, Haykal Hospital, Tripoli, \\ Lebanon; ' ${ }^{6}$ Department of Hematology and Oncology, Hammoud Hospital University Medical Center, Saida, Lebanon; ${ }^{7}$ Roche Lebanon SARL, Beirut, \\ Lebanon; ${ }^{8}$ Department of Hematology and Oncology, Hôtel-Dieu de France University Hospital, Beirut, Lebanon
}

Correspondence: Ali Shamseddine, Department of Internal Medicine, American University of Beirut Medical Center, Beirut, Lebanon, Tel +96 | | 374374, Fax+96| | 3708 |4, Email as04@aub.edu.lb

Purpose: When combined with chemotherapy, bevacizumab improves progression-free survival (PFS) in metastatic colorectal cancer (mCRC). This observational trial was designed to assess the safety and efficacy of bevacizumab plus first-line chemotherapy in a realworld setting in Lebanon.

Patients and Methods: A non-interventionaL multicenter study of first-LIne AVastin ${ }^{\circledR}$ (bevacizumab) in combination with chEmotherapy in patients with metastatic colorectal cancer (LLIVE) is a multicenter, prospective, Lebanon-based, observational study that enrolled mCRC patients who received first-line bevacizumab plus chemotherapy combination. The primary end point of the study was PFS. Secondary endpoints comprised the overall response rate (ORR) and the safety and tolerability of bevacizumab.

Results: A total of 196 patients were enrolled between July 2010 and August 2013. The median duration of follow-up was 11 months. Median duration of bevacizumab treatment was 4 months with FOLFOX being the chiefly chemotherapy regimen used in the first-line setting (26\%). Median PFS was 8.22 months (95\% confidence interval (CI): 7.005-9.443). The ORR was 50.3\% (complete response $7.5 \%$, partial response $42.8 \%$ ). The most common adverse event encountered was hypertension (28\%) followed by epistaxis (4.8\%), diarrhea (4\%), anemia (4\%) and headache (4\%). Grade 3/4 adverse events occurred in $15.2 \%$ of patients.

Conclusion: The trial further substantiated the efficacy and safety of bevacizumab and chemotherapy in the first-line treatment of mCRC patients in Lebanon.

Keywords: metastatic colorectal cancer, bevacizumab, FOLFOX, XELOX

\section{Introduction}

Worldwide, colorectal cancer (CRC) is a deadly disease if not diagnosed early and ranks third and second among cancers in males and females, respectively. According to the Lebanese National Cancer Registry report in 2016, ${ }^{1} \mathrm{CRC}$ ranks fourth among males and second among females with an age adjusted incidence of 23/100,000 and 19.7/100,000, respectively. Even though the incidence rates of CRC remain high, CRC treatment modalities such as polyps' removal and early detection methods have proven efficacious in decreasing CRC mortality. ${ }^{2}$ The implementation of improved screening tests may have been the reason behind the increased incidence but have substantially influenced mortality on the long run attributed to the elimination of pre-cancerous or un-metastasized polyps. ${ }^{3}$ However, in stage IV or metastatic disease, the survival rate for metastatic CRC remains low with only $12 \%$ surviving at 5 years. ${ }^{3}$ 
The three active conventional chemotherapy agents for mCRC are fluoropyrimidines, irinotecan and oxaliplatin. The most widely used cytotoxic backbone involves double-agent chemotherapy (FOLFOX/XELOX or FOLFIRI), while triple-agent chemotherapy (FOLFOXIRI), although achieving better progression-free survival (PFS), response rate (RR) and OS than FOLFIRI in some trials, ${ }^{4,5}$ is only reserved to patients who can tolerate such an aggressive regimen. 5-FU/ LV or capecitabine, which have been shown to be inferior to FOLFOX ${ }^{6-8}$ and FOLFIRI ${ }^{9,10}$ in terms of OS (with FOLFIRI regimen), PFS and RR, are still a treatment of choice in patients who cannot tolerate treatment with oxaliplatin and irinotecan. The humanized monoclonal vascular endothelial growth factor (VEGF) antibody bevacizumab has improved PFS in the first and second-line treatment of mCRC. ${ }^{11-15}$ The main adverse events (AEs) associated with the use of bevacizumab involved hypertension, proteinuria, impaired wound healing, gastrointestinal perforation, hemorrhage and arterial thromboembolism which were mainly mild or moderate in severity. ${ }^{12-14}$ Some observational cohort studies also backed the efficacy and safety findings of bevacizumab. ${ }^{16-18}$

The non-interventionaL multicenter study of first-LIne AVastin ${ }^{\circledR}$ (bevacizumab) in combination with chEmotherapy in patients with metastatic colorectal cancer (LLIVE) observational study evaluated the safety and efficacy of bevacizumab in the first-line treatment of mCRC patients in Lebanon.

\section{Materials and Methods}

\section{Patients and Regulatory Issues}

Patients with $\mathrm{mCRC}$ who were $\geq 18$ years and who received bevacizumab as first-line treatment were included. A written informed consent to participate was obtained from each patient prior to recruitment. Women with a childbearing potential were enrolled only if they were using effective contraception. Patients with liver-only metastasis who were fit for surgery underwent surgical resection. Exclusion criteria included contraindication to receive bevacizumab according to the local labeling, patients receiving bevacizumab as a foreseen peri-operative metastasectomy treatment, participation in a study within 30 days prior to enrollment, having any other primary cancer, concomitant treatment with other biologics, and suffering from malignant diseases in the past 5 years, except for basal cell carcinoma. This study was conducted in accordance with the Good Pharmaco-epidemiology Practice as well as recommendations for non-interventional trials and principles of epidemiology studies. The study was also approved by the ethics committees and/or institutional review boards (IRB) of each of the following participating institutions: American University of Beirut Medical Center, Mount Lebanon Hospital, Saint-Joseph University, Middle East Institute of Health, Haykal Hospital, Hammoud Hospital University Medical Center, Hôtel-Dieu de France University Hospital, Ain Wazein Hospital, Al Rassoul Al Aazam hospital, Saint Joseph Hospital, Notre Damme De Secours and Beirut Governmental Hospital. All research was conducted according to the above IRB approved method in accordance with the declaration of Helsinki.

\section{Study Design}

This was a prospective, multicenter, non-interventional study. The primary endpoint was to estimate PFS and overall response rate (ORR). Secondary endpoints involved describing the treatment regimens employed and assessing the safety of bevacizumab in patients with mCRC from 12 centers in Lebanon; American University of Beirut Medical Center, Mount Lebanon Hospital, Saint-Joseph University, Middle East Institute of Health, Haykal Hospital, Hammoud Hospital University Medical Center, Hôtel-Dieu de France University Hospital, Ain Wazein Hospital, Al Rassoul Al Aazam hospital, Saint Joseph Hospital, Notre Damme De Secours and Beirut Governmental Hospital. Data required for the study was obtained from the medical charts of patients at inclusion and every 3 months until the end of treatment and then for a total of 6 months after the last dose of bevacizumab.

\section{Assessments}

From the medical chart of each patient, information pertaining to demographics, disease characteristics, medical history, treatment modalities, comorbidities and laboratory values were obtained. During the follow-up visits, tumor and safety assessment and modifications to chemotherapy and bevacizumab schedules were collected. Tumor assessment was based on CT-scan using Response Evaluation Criteria in Solid Tumors (RECIST) criteria version 1.1. Investigators performed the RECIST assessment according to standard clinical practice and recorded the result in the case report form. ORR 
included patients who had a partial or complete response (PR and CR respectively) to therapy, and did not include stable disease (SD). It was computed at visit 1 and at the final visit. Disease control rate (DCR) was determined by computing the percentage of patients who had CR, PR and SD. PFS was calculated from the time bevacizumab was initiated to disease progression or death, whichever occurred before. All clinical adverse events (AEs) encountered during the clinical study were reported on the AE page of the case report form. Intensity of adverse events was graded on a threepoint scale (mild, moderate, severe) and reported in detail. Moreover, the relationship of the adverse event to the treatment was also assessed (probable, possible, remote, unrelated).

\section{Study Size and Statistical Analysis}

The primary endpoint of this study was the PFS. Assuming an expected progression of $35 \%$ of mCRC patients using Bevacizumab within 10 months as opposed to $56 \%$ of patients on chemotherapy alone, and considering a confidence interval (CI) of $95 \%$ and an acceptable difference up to 0.06 between the sample proportion and the population proportion, the sample size has been set at 243 patients. ${ }^{14}$

Descriptive statistics were employed to describe patient and disease characteristics and treatment modalities. The disease characteristics were compared across risk factors and demographic characteristics using Pearson chi-square for categorical variables, and ANOVA for continuous variables. Dependent samples t-tests (Mc-Nemar) were also performed to assess the change in disease characteristics across time. The median PFS was estimated using the Kaplan-Meier method along with $95 \%$ CI. A two-sided p-value $<0.05$ was considered statistically significant. Missing data were not replaced. Statistical analyses were performed using IBM SPSS, version 20.0 for Windows release (IBM Corp. Released 2011. IBM SPSS Statistics for Windows, Version 20.0. Armonk, NY: IBM Corp.)

\section{Results}

\section{Patients and Diseases Characteristics}

Between July 2010 and August 2013, 196 patients were registered at 12 centers in Lebanon. Baseline characteristics for all the patients included are shown in Table 1. Patients' mean age was $61.7 \pm 13.7$ years; $50 \%$ of patients were male, 93.4\% had an ECOG-PS score of 0 or 1 and $7.7 \%$ had family history of CRC.

The colon constituted the most common site of primary tumor location (82.7\%). Seventy-three percent and $65.8 \%$ had synchronous and liver metastases, respectively; $19.4 \%$ underwent surgery, $23.5 \%$ received neo-adjuvant treatment and $6.1 \%$ received adjuvant treatment before initiation of bevacizumab. Liver-only metastasis occurred in 96 patients out of which 53 (55\%) underwent surgical resection. Bevacizumab dosing was given at $5 \mathrm{mg} / \mathrm{kg}$ every 2 weeks in 149 patients, and at $7.5 \mathrm{mg} / \mathrm{kg}$ every 3 weeks in 46 patients specifically those receiving XELIRI or XELOX. Only one patient received $10 \mathrm{mg} / \mathrm{kg}$ every 3 weeks which was off label at the discretion of his/her treating physician.

The majority of patients (76\%) received $5 \mathrm{mg} / \mathrm{kg}$ of bevacizumab every 2 weeks, for a median duration of 4 months (range: 0-31) (Table 2). Dose changes were reported in 10.2\% (9 patients had permanent dose reduction while 11 patients had temporary dose reduction due to toxicity), permanent discontinuation took place in $67.9 \%$ of cases. Main reasons for discontinuation were disease progression (45.4\%) and adverse events (7.7\%). Oxaliplatin-based therapies were the most common. FOLFOX (folinic acid-fluorouracil-oxaliplatin, 32.6\%), XELOX (capecitabine-oxaliplatin, 22.5\%) and FOLFIRI (8.2\%) were the most prescribed regimens (Table 2).

\section{Efficacy}

At a median follow-up of 11 months (range: 1-28), PFS was 8.22 months (95\% CI: 7.005-9.443) in 189 patients (Figure 1). Median OS was not reached and the reported mean OS was 20.6 months (95\% CI: 18.8-22.5). Data on tumor response to treatment were available in 173 patients. The response rate was $50.3 \% ; 7.5 \%$ CR and $42.8 \%$ PR. Stable disease was seen $33.5 \%$ while progressive disease occurred in $16.2 \%$. Kaplan Meier curves were plotted to identify any differences in PFS among the different chemotherapy regimens, RAS, and tumor sidedness. No differences were shown by side and chemotherapy regimens. Significant difference was shown in median PFS for wild-type KRAS (6 months) versus mutant KRAS (9 months) ( $\mathrm{P}=0.22$ ) (Figure 2). 
Table I Patients' Biographical and Clinical Data

\begin{tabular}{|c|c|}
\hline & Total $(n=196)$ \\
\hline \multicolumn{2}{|l|}{ Gender, n (\%) } \\
\hline Male & $98(50.0)$ \\
\hline Age, y, mean (SD) & $61.7(13.7)$ \\
\hline \multicolumn{2}{|l|}{ ECOG performance status $\mathrm{n}(\%)$} \\
\hline $0-1$ & $183(93.4)$ \\
\hline $2-3$ & $13(6.6)$ \\
\hline \multicolumn{2}{|l|}{ Primary tumor } \\
\hline Time between initial diagnosis and inclusion (mo), median (range) & I (0-173) \\
\hline \multicolumn{2}{|l|}{ Primary tumor location, n (\%) } \\
\hline Left colon & $99(50.5)$ \\
\hline Right colon & $63(32.1)$ \\
\hline Rectum & $34(17.4)$ \\
\hline \multicolumn{2}{|l|}{ Previous tumor treatment, $\mathrm{n}(\%)$} \\
\hline Surgery of the primary tumor & $38(19.4)$ \\
\hline Neoadjuvant treatment & $46(23.5)$ \\
\hline Adjuvant treatment & $12(6.1)$ \\
\hline \multicolumn{2}{|l|}{ Metastatic disease } \\
\hline \multicolumn{2}{|l|}{ Type of metastasis, $n(\%)$} \\
\hline Synchronous & $143(73.0)$ \\
\hline Metachronous & $53(27.0)$ \\
\hline \multicolumn{2}{|l|}{ Metastatic sites, n (\%) } \\
\hline Liver & $129(65.8)$ \\
\hline Lung & $54(27.6)$ \\
\hline Peritoneum & $85(23.8)$ \\
\hline Brain & $3(1.5)$ \\
\hline Other & $79(40.3)$ \\
\hline \multicolumn{2}{|l|}{ Number of metastatic sites, $\mathrm{n}(\%)$} \\
\hline 1 & $119(60.7)$ \\
\hline 2 or more & $77(39.3)$ \\
\hline \multicolumn{2}{|l|}{ Biomarkers, n (\%) } \\
\hline K-RAS Mutant & $56(28.6)$ \\
\hline KRAS wild-type & $55(28.1)$ \\
\hline Unavailable & $85(43.4)$ \\
\hline CEA marker (ng/L), mean (SD) & $262.5(1017.3)$ \\
\hline
\end{tabular}


Table 2 Characteristics of First-Line Bevacizumab and Combined Chemotherapy

\begin{tabular}{|c|c|}
\hline & Total $(n=196)$ \\
\hline \multicolumn{2}{|l|}{ Bevacizumab regimen, $\mathrm{n}(\%)$} \\
\hline $5 \mathrm{mg} / \mathrm{kg} / 2 \mathrm{wk}$ & 149 \\
\hline $7.5 \mathrm{mg} / \mathrm{kg} / 3 \mathrm{wk}$ & 46 \\
\hline $10 \mathrm{mg} / \mathrm{kg} / 3 \mathrm{wk}$ & I \\
\hline Treatment duration (mo), median (range) & 4 months (Range: $0-3 \mathrm{I}$ ) \\
\hline At least I change in dosing, $n(\%)$ & $20(10.2)$ \\
\hline At least I temporary discontinuation, $n(\%)$ & $61(31.1)$ \\
\hline Permanent discontinuation, n (\%) & $133(67.9)$ \\
\hline \multicolumn{2}{|l|}{ Reason for permanent discontinuation $(n=133)$} \\
\hline Progression & $89(45.4)$ \\
\hline Adverse event & $15(7.7)$ \\
\hline Loss to follow up & $29(14.8)$ \\
\hline \multicolumn{2}{|l|}{ Combined chemotherapy, n (\%) } \\
\hline Folfox & $64(32.6)$ \\
\hline Xelox & $44(22.5)$ \\
\hline Folfiri & $16(8.2)$ \\
\hline XELIRI & II (5.6) \\
\hline Oxaliplatin & II (5.6) \\
\hline Capecitabine & $5(2.6)$ \\
\hline Irinotecan & $4(2)$ \\
\hline 5-FU leucovorin & $5(2.5)$ \\
\hline Oxaliplatin/irinotecan & $3(1.5)$ \\
\hline FOLFOXIRI & $2(1.0)$ \\
\hline Not reported & $31(15.8)$ \\
\hline
\end{tabular}

\section{Tolerability}

A total of 125 adverse events were reported in relation to bevacizumab treatment (Table 3). Most adverse events $(84.8 \%)$ were grade $1 / 2$. Ten (8\%) were grade 4 and required hospitalization. Two (1.6\%) were grade 5 infarctions which resulted in death. The most common adverse event encountered was hypertension (28\%) followed by epistaxis (4.8\%), diarrhea (4\%), anemia (4\%) and headache (4\%).

\section{Discussion}

This study thoroughly described treatment modalities employed in Lebanon and assessed the efficacy and safety of firstline bevacizumab. The median PFS reported in this study was 8.22 months which is similar to that reported by other observational studies (8.7 months in ACORN, 9.9 months in BRiTE, 10.8 months in BEAT, 10.2 months in ARIES), 


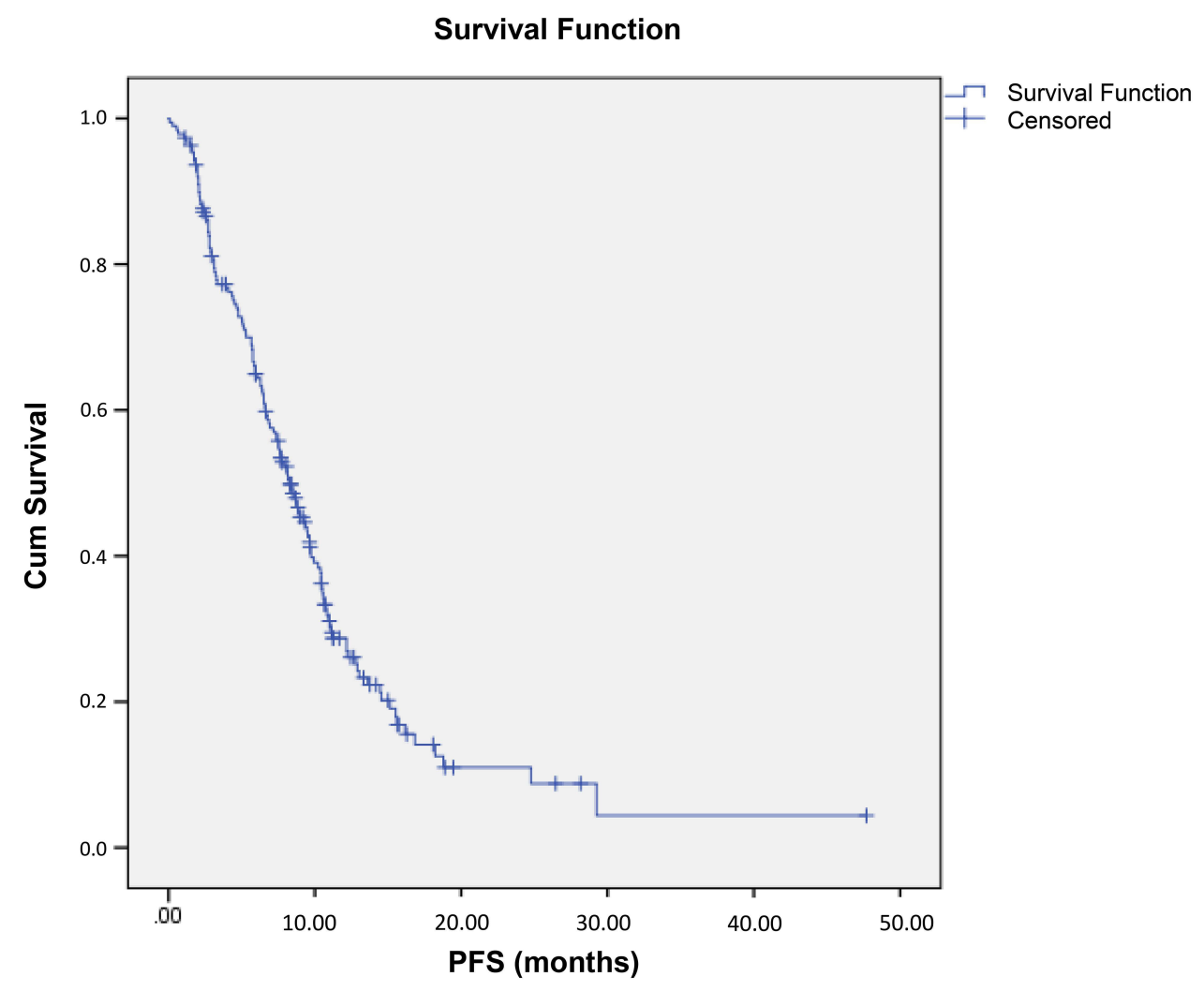

Figure I Kaplan-Meier survival curve of progression-free survival in metastatic colorectal cancer patients receiving first-line chemotherapy-bevacizumab.

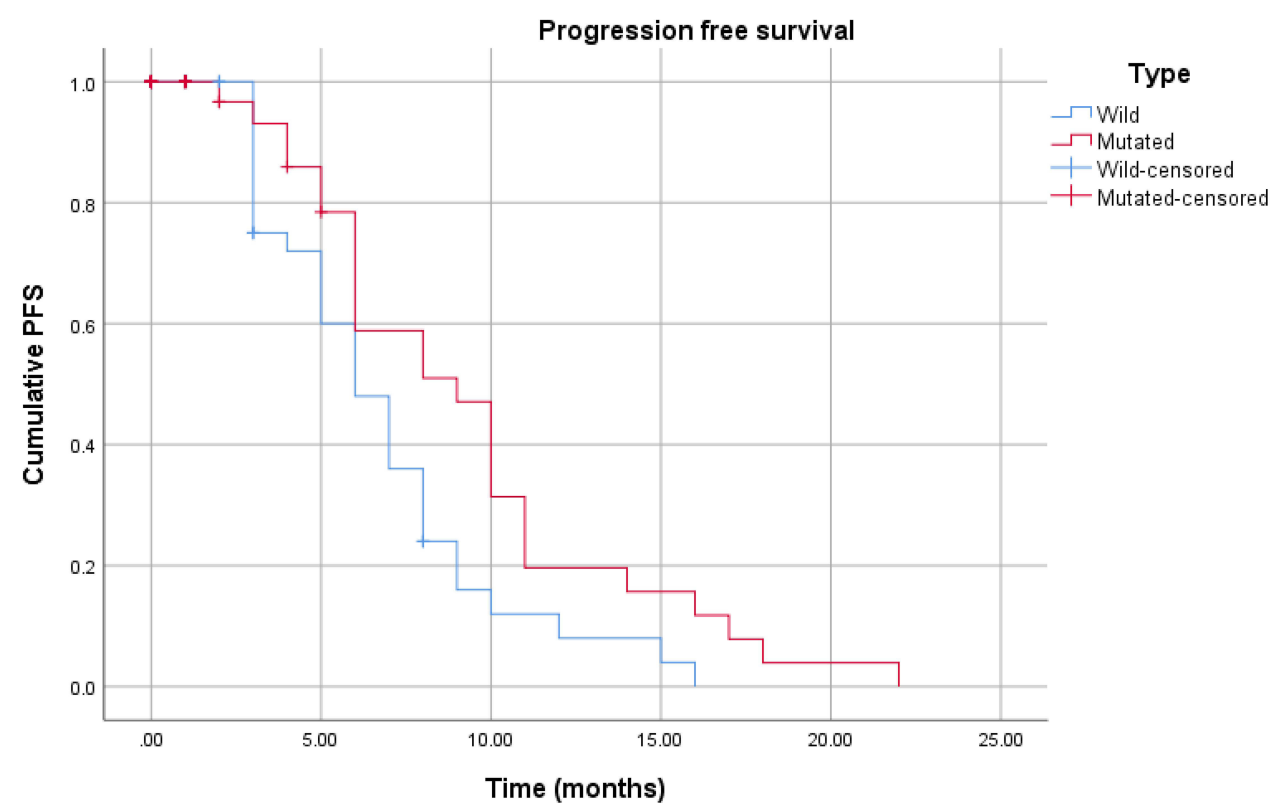

Figure 2 Kaplan-Meier curve of progression-free survival in metastatic colorectal cancer patients with wild-type KRAS versus mutated KRAS.

though shorter. ${ }^{16-19}$ Median OS was not reached since patients were only followed up for a period of 11 months and OS was not the objective of the LLIVE observational trial.

Data from the 173 evaluable patients in this observational study revealed an overall ORR of $50.3 \%$, which is similar to that reported in the ITACa randomized trial ${ }^{20}$ but higher than previously reported in other randomized trials ${ }^{13,14}$ and 
Table 3 The Reported Adverse Events from Bevacizumab Treatment

\begin{tabular}{|c|c|c|c|c|c|}
\hline & Grade I & Grade 2 & Grade 3 & Grade 4 & Total \\
\hline Diarrhea & 4 & I & & & 5 \\
\hline Nausea & 3 & & I & & 4 \\
\hline Vomiting & 2 & & & & 2 \\
\hline Abdominal pain & 3 & I & & & 4 \\
\hline Rectal bleeding & 1 & & & 2 & 3 \\
\hline Subcapsular bleeding & 1 & & & & 1 \\
\hline Hypertension & 10 & 24 & I & & 35 \\
\hline Aortic thrombosis & 1 & & & & 1 \\
\hline Left leg deep vein thrombosis & & I & & & 1 \\
\hline Proteinuria & I & I & & & 2 \\
\hline Neuropathy & 1 & & & & 1 \\
\hline Left hemiparesis & & I & & & 1 \\
\hline Constipation & 1 & & & & 1 \\
\hline Dyspnea & 3 & & & & 3 \\
\hline Chest pain & 3 & & & & 3 \\
\hline Cough & 2 & & & & 2 \\
\hline Fatigue & 2 & & & & 2 \\
\hline Asthenia & 1 & & I & & 2 \\
\hline Anemia & 4 & I & & & 5 \\
\hline Fever & & I & & & 1 \\
\hline Headache & 1 & 4 & & & 5 \\
\hline Rash & 2 & & & & 2 \\
\hline Acne & 1 & & & & 1 \\
\hline Epistaxis & 5 & & & I & 6 \\
\hline Hemoptysis & & I & & I & 2 \\
\hline Intestinal perforation & & & & 2 & 2 \\
\hline Neutropenia & & 1 & 3 & & 4 \\
\hline Thrombocytopenia & 2 & 2 & & & 4 \\
\hline Leucocytopenia & & I & & & I \\
\hline Gastroenteritis & & I & 2 & & 3 \\
\hline Mucositis & 1 & & I & & 2 \\
\hline Darkening skin & 2 & & & & 2 \\
\hline Digestive hemorrhage & & & & 2 & 2 \\
\hline
\end{tabular}

(Continued) 
Table 3 (Continued).

\begin{tabular}{|c|c|c|c|c|c|}
\hline & Grade I & Grade 2 & Grade 3 & Grade 4 & Total \\
\hline Cerebral hemorrhage & I & & & & I \\
\hline Urosepsis & & & & I & I \\
\hline Hallucinations & I & & & & I \\
\hline Pneumonia & & I & & & I \\
\hline Rectovaginal fistula & & & & I & 1 \\
\hline Enterocutaneous fistula & & I & & & 1 \\
\hline Renal failure & & I & & & 1 \\
\hline Decreased food intake & I & & & & I \\
\hline Healing problem & & I & & & 1 \\
\hline Odynophagia & I & & & & 1 \\
\hline Total & 61 & 45 & 9 & 10 & 125 \\
\hline
\end{tabular}

lower than that reported by a German observational study. ${ }^{21}$ One reason behind the higher ORR reported could be that RECIST assessment was based on investigator assessment not independent review. The importance of observational cohort studies lies in their ability to collect more data on the real-world treatment patterns and treatment-related adverse events and to check for the occurrence of any new safety indicator. Previous studies have identified several adverse events associated with the use of bevacizumab which were generally similar to those reported in the present study. In LLIVE, two patients (1.6\%) experienced a gastrointestinal perforation similar to those seen in ACORN (2.2\%), BRiTE (1.9\%), BEAT (2\%) and ARIES (1.2\%). ${ }^{16-19}$ Hypertension was the most frequently reported adverse event in LLIVE (28\%) which was more common than in ACORN (11.3\%), BRiTE (22\%), BEAT (5.3\%) and ARIES (8.6\%). ${ }^{16-19}$ Hemorrhage and arterial thromboembolic events are two adverse events which are of concern to oncologists. In LLIVE, grade 4 digestive hemorrhage occurred in 1.6\% and grade 4 rectal bleeding occurred in 1.6\% which is similar to rates of bleeding reported by other observational studies. Grade 5 events leading to death occurred in $1.6 \%$ in LLIVE which is less than rates reported in ACORN (2.9\%), BEAT (3\%) and BRiTE (2.1\%). ${ }^{15-17}$

Limitations of this study include its observational design specifically the bias deployed in the selection of patients for a certain treatment, in assessing response to treatment and in determining progression rate. Also, since data were obtained from individual centers, there was no autonomous review of scans. Moreover, there was no detailed description to the treatment strategies used following first-line therapy. Moreover, as 243 patients were required in the study protocol but only 196 patients were enrolled could have had some impact on the results. Main reasons behind not reaching the required number were related to the Lebanese population which is relatively a small one as well as the limited time frame of the study. Finally, the results of LLIVE are utterly from Lebanese mCRC patients, a fact which restricts the generalizability of the results.

\section{Conclusion}

In conclusion, this trial which is based in Lebanon reported a safety and efficacy profile similar to that reported in other observational studies around the world assessing the impact of first-line chemotherapy with bevacizumab for the treatment of mCRC patients. The results shown offer further validation to the positive impact of bevacizumab plus chemotherapy on PFS in mCRC patients, specifically in those with wild-type KRAS.

\section{Acknowledgments}

There are no researchers to be included in this section. 


\section{Disclosure}

Dr Layal Maatouk reports being an employee of Roche Lebanon SARL, outside the submitted work. The authors report no other conflicts of interest in this work.

\section{References}

1. Incidence rates by site.MOPH; 2016. https://www.moph.gov.lb/en/Pages/8/19526/national-cancer-registry. Accessed November $29,2021$.

2. Edwards BK, Ward E, Kohler BA, et al. Annual report to the nation on the status of cancer, 1975-2006, featuring colorectal cancer trends and impact of interventions (risk factors, screening, and treatment) to reduce future rates. Cancer. 2010;116(3):544-573. doi:10.1002/cncr.24760

3. Arnold M, Sierra MS, Laversanne M, Soerjomataram I, Jemal A, Bray F. Global patterns and trends in colorectal cancer incidence and mortality. Gut. 2017;66(4):683-691. doi:10.1136/gutjnl-2015-310912

4. Falcone A, Ricci S, Brunetti I, et al. Phase III trial of infusional fluorouracil, leucovorin, oxaliplatin, and irinotecan (FOLFOXIRI) compared with infusional fluorouracil, leucovorin, and irinotecan (FOLFIRI) as first-line treatment for metastatic colorectal cancer: the Gruppo Oncologico Nord Ovest. J Clin Oncol. 2007;25(13):1670-1676. doi:10.1200/JCO.2006.09.0928

5. Masi G, Vasile E, Loupakis F, et al. Randomized trial of two induction chemotherapy regimens in metastatic colorectal cancer: an updated analysis. J Natl Cancer Inst. 2011;103(1):21-30. doi:10.1093/jnci/djq456

6. de Gramont A, Figer A, Seymour M, et al. Leucovorin and fluorouracil with or without oxaliplatin as first-line treatment in advanced colorectal cancer. J Clin Oncol. 2000;18(16):2938-2947. doi:10.1200/JCO.2000.18.16.2938

7. Grothey A, Sargent D. Overall survival of patients with advanced colorectal cancer correlates with availability of fluorouracil, irinotecan, and oxaliplatin regardless of whether doublet or single-agent therapy is used first line. J Clin Oncol. 2005;23(36):9441-9442. doi:10.1200/ JCO.2005.04.4792

8. Giacchetti S, Perpoint B, Zidani R, et al. Phase III multicenter randomized trial of oxaliplatin added to chronomodulated fluorouracil-leucovorin as first-line treatment of metastatic colorectal cancer. J Clin Oncol. 2000;18(1):136-147. doi:10.1200/JCO.2000.18.1.136

9. Douillard JY, Cunningham D, Roth AD, et al. Irinotecan combined with fluorouracil compared with fluorouracil alone as first-line treatment for metastatic colorectal cancer: a multicentre randomised trial. Lancet. 2000;355(9209):1041-1047. doi:10.1016/S0140-6736(00)02034-1

10. Saltz LB, Cox JV, Blanke C, et al. Irinotecan plus fluorouracil and leucovorin for metastatic colorectal cancer. Irinotecan Study Group. $N$ Engl $J$ Med. 2000;343(13):905-914. doi:10.1056/NEJM200009283431302

11. Kabbinavar F, Hurwitz HI, Fehrenbacher L, et al. Phase II, randomized trial comparing bevacizumab plus fluorouracil (FU)/leucovorin (LV) with FU/LV alone in patients with metastatic colorectal cancer. $J$ Clin Oncol. 2003;21(1):60-65. doi:10.1200/JCO.2003.10.066

12. Kabbinavar FF, Schulz J, McCleod M, et al. Addition of bevacizumab to bolus fluorouracil and leucovorin in first-line metastatic colorectal cancer: results of a randomized Phase II trial. J Clin Oncol. 2005;23(16):3697-3705. doi:10.1200/JCO.2005.05.112

13. Hurwitz H, Fehrenbacher L, Novotny W, et al. Bevacizumab plus irinotecan, fluorouracil, and leucovorin for metastatic colorectal cancer. $N$ Engl $J$ Med. 2004;350(23):2335-2342. doi:10.1056/NEJMoa032691

14. Saltz LB, Clarke S, Díaz-Rubio E, et al. Bevacizumab in combination with oxaliplatin-based chemotherapy as first-line therapy in metastatic colorectal cancer: a randomized phase III study. J Clin Oncol. 2008;26(12):2013-2019. doi:10.1200/JCO.2007.14.9930

15. Giantonio BJ, Catalano PJ, Meropol NJ, et al. Bevacizumab in combination with oxaliplatin, fluorouracil, and leucovorin (FOLFOX4) for previously treated metastatic colorectal cancer: results from the Eastern Cooperative Oncology Group Study E3200. J Clin Oncol. 2007;25 (12):1539-1544. doi:10.1200/JCO.2006.09.6305

16. Van Cutsem E, Rivera F, Berry S, et al. Safety and efficacy of first-line bevacizumab with FOLFOX, XELOX, FOLFIRI and fluoropyrimidines in metastatic colorectal cancer: the BEAT study. Ann Oncol. 2009;20(11):1842-1847. doi:10.1093/annonc/mdp233

17. Kozloff M, Yood MU, Berlin J, et al. Clinical outcomes associated with bevacizumab-containing treatment of metastatic colorectal cancer: the BRiTE observational cohort study. Oncologist. 2009;14(9):862-870. doi:10.1634/theoncologist.2009-0071

18. Hurwitz HI, Bekaii-Saab TS, Bendell JC, et al. Safety and effectiveness of bevacizumab treatment for metastatic colorectal cancer: final results from the Avastin $\left({ }^{\circledR}\right)$ Registry - Investigation of Effectiveness and Safety (ARIES) observational cohort study. Clin Oncol. 2014;26(6):323-332. doi:10.1016/j.clon.2014.03.001

19. Khakoo S, Chau I, Pedley I, et al. ACORN: observational study of bevacizumab in combination with first-line chemotherapy for treatment of metastatic colorectal cancer in the UK. Clin Colorectal Cancer. 2019;18(4):280-291.e285. doi:10.1016/j.clcc.2019.07.003

20. Passardi A, Nanni O, Tassinari D, et al. Effectiveness of bevacizumab added to standard chemotherapy in metastatic colorectal cancer: final results for first-line treatment from the ITACa randomized clinical trial. Ann Oncol. 2015;26(6):1201-1207. doi:10.1093/annonc/mdv130

21. Stein A, Petersen V, Schulze M, et al. Bevacizumab plus chemotherapy as first-line treatment for patients with metastatic colorectal cancer: results from a large German community-based observational cohort study. Acta Oncol. 2015;54(2):171-178. doi:10.3109/0284186X.2014.961649

Biologics: Targets and Therapy

Dovepress

\section{Publish your work in this journal}

Biologics: Targets and Therapy is an international, peer-reviewed journal focusing on the patho-physiological rationale for and clinical application of Biologic agents in the management of autoimmune diseases, cancers or other pathologies where a molecular target can be identified. This journal is indexed on PubMed Central, CAS, EMBase, Scopus and the Elsevier Bibliographic databases. The manuscript management system is completely online and includes a very quick and fair peer-review system, which is all easy to use. Visit http://www.dovepress.com/ testimonials.php to read real quotes from published authors.

Submit your manuscript here: https://www.dovepress.com/biologics-targets-and-therapy-journal 